\title{
Composición, estructura e importancia ecológica de las Moraceae en un bosque residual de Ucayali, Perú
}

\author{
Composition, structure and ecological importance of the Moraceae \\ in a residual forest of Ucayali, Peru
}

\author{
Fred C. Ramírez ${ }^{1, \star}$, Gumercindo A. Castillo ${ }^{1}$, Ymber Flores², \\ Octavio F. Galván ${ }^{1}$, Luisa Riveros ${ }^{1}$ y Lyanna H. Sáenz ${ }^{3}$
}

\begin{abstract}
Recibido: 15 agosto 2021 | Aceptado: 21 diciembre 2021 | Publicado en línea: 29 diciembre 2021 Citación: Ramírez,FC; Castillo, GA; Flores, Y; Galván, OF; Riveros, L; Sáenz,LH.2021.Composición, estructura e importancia ecológica de las Moraceae en un bosque residual de Ucayali, Perú. Revista Forestal del Perú 36(2): 247-260. DOI: http://dx.doi.org/10.21704/rfp.v36i2.1801
\end{abstract}

\begin{abstract}
Resumen
Las especies de la familia Moraceae tienen gran importancia económica, medicinal y ecológica en la Amazonía; sin embargo, existen escasos estudios sobre su diversidad y dinámica poblacional en los bosques residuales. El objetivo fue determinar la composición, estructura e importancia ecológica de las Moraceae en un bosque residual. El método aplicado fue el descriptivo y consistió en establecer 16 parcelas de $20 \mathrm{~m} \times 50 \mathrm{~m}$ (0,10 ha), en un bosque residual de la subestación Alexánder von Humboldt, del Instituto Nacional de Innovación Agraria-INIA, Pucallpa, departamento de Ucayali, donde se evaluaron los individuos de hábito arbóreo o hemiepífito, con DAP $\geq 2,50 \mathrm{~cm}$. La composición florística estuvo representada por 33 especies, distribuidas en 12 géneros; se encontró cinco especies no registradas para Ucayali. Estructuralmente, la familia estuvo representada por 138 individuos/ha con una distribución horizontal semejante a una "J" invertida irregular, sin embargo, existieron diferentes estructuras horizontales entre especies. Se determinó que el 85\% de las especies se encontraban en la clase diamétrica I ( 2,50 a 9,99 cm), siendo la más abundante Pseudolmedia laevis (Ruiz \& Pav.) J.F. Macbr. (41,88 individuos/ha); y las más dominantes fueron Brosimum utile (Kunth) Oken $\left(1,71 \mathrm{~m}^{2} / \mathrm{ha}\right)$ y Brosimum alicastrum subsp. bolivarense (Pittier) C.C.Berg $\left(0,90 \mathrm{~m}^{2} /\right.$ ha). Asimismo, P. laevis y B. utile fueron las de mayor importancia ecológica. La información de la presente investigación permitirá establecer una línea base, que puede ser utilizada para proponer el manejo de las Moraceae en bosques residuales del mismo ámbito de estudio.
\end{abstract}

Palabras clave: bosque residual, abundancia, dominancia, IVI, nuevos registros

\footnotetext{
${ }^{1}$ Universidad Nacional Intercultural de la Amazonía (UNIA), Pucallpa, Perú.

${ }^{2}$ Instituto Nacional de Innovación Agraria (INIA), Pucallpa, Perú.

${ }^{3}$ Universidade do Estado do Amazonas (UEA), Manaus, Brasil.

*Autor de Correspondencia: fredc.ramirez.g@gmail.com
} 


\begin{abstract}
The species of the Moraceae family have great economic, medicinal and ecological importance in the Amazon; however, there are few studies on their diversity and population dynamics in residual forests. The objective was to determine the composition, structure, and ecological importance of the Moraceae in a residual forest. The method applied was descriptive and consisted of establishing 16 plots of $20 \mathrm{~m} \times 50 \mathrm{~m}(0,10 \mathrm{ha})$, in a residual forest of the substation Alexander von Humboldt, from the National Institute of Agricultural Innovation-INIA, Pucallpa, department of Ucayali, where individuals of arboreal or hemiepiphytic habit were evaluated, with $\mathrm{DBH} \geq 2,50 \mathrm{~cm}$. The floristic composition was represented by 33 species, distributed in 12 genera; five unrepealed species were found for Ucayali. Structurally, the family was represented by 138 individuals/ha with a horizontal distribution similar to an irregular inverted "J", however, there were different horizontal structures between species. It was determined that $85 \%$ of the species were in diametric class I (2,50 to 9,99 $\mathrm{cm}$ ), the most abundant being Pseudolmedia laevis (Ruiz \& Pav.) J.F. Macbr. (41,88 individuals/ha); and the most dominant were Brosimum utile (Kunth) Oken $\left(1,71 \mathrm{~m}^{2} / \mathrm{ha}\right)$ and Brosimum alicastrum subsp. bolivariense (Pittier) C.C.Berg $\left(0,90 \mathrm{~m}^{2} / \mathrm{ha}\right)$. Likewise, P. laevis and B. utile were the most ecologically important. The information in this investigation will make it possible to establish a baseline, which can be used to propose the management of the Moraceae in residual forests of the same field of study.
\end{abstract}

Key words: residual forest, abundance, dominance, IVI, new records

\section{Introducción}

El Perú cuenta con una riqueza arbórea de 4618 especies, agrupadas en 148 familias (Vásquez et al. 2018), lo cual representa una oportunidad incomparable y una prioridad urgente para la realización de investigaciones florísticas. Además, es considerado como uno de los países biológicamente más diversos en el mundo (MINAM 2014). Sin embargo, grandes áreas forestales permanecen inexploradas (Honorio y Reynel 2003), y los registros aún son incompletos y fragmentados (Flores 2018, Vásquez et al. 2018). Las Moraceae presentan una considerable abundancia y riqueza de especies (Mostacedo et al. 2006, Calvi 2013, García 2014), lo cual influye para que sea considerada de importancia ecológica en los distintos bosques de la Amazonía (Neill y Killeen 1991, Nebel et al. 2000, Cardona-Peña et al. 2005, Araujo-Murakami et al. 2005, Marcelo-Peña y Reynel 2014, Mena-Mosquera et al. 2020). Recientes estudios encontraron que Brosimum utile (Kunth) Oken es la especie de mayor peso ecológico, a $107 \mathrm{msnm}$ en Colombia (Mena-Mosquera et al. 2020); mientras que, en Ecuador, Ficus cuatracasana Dugand domina el espacio horizontal, entre 601 a $1000 \mathrm{msnm}$
(García 2019). En el Perú la familia Moraceae está representada por 19 géneros, 128 especies, siendo el género Ficus el más diverso con 102 especies y 20 subespecies (Brako y Zarucchi, 1993; Ulloa Ulloa et al. 2004, 2017; Vásquez et al. 2018), lo cual ha sido complementado con el descubrimiento de nuevas especies y nuevos reportes para la flora peruana (Berg y Homeier 2010, Mitidieri et al. 2020), que en conjunto agrupan todos los nombres reconocidos hasta la fecha; mientras que, para la región Ucayali existe un total de 58 especies y 6 subespecies (Flores 2018).

Las Moraceae son de importancia económica principalmente por el valor de su madera (Reynel et al. 2016, Flores 2018, SERFOR 2020), siendo las especies más utilizadas, en la transformación primaria, Brosimum utile subsp. ovatifolium (Ducke) C.C. Berg "Panguana", $M a-$ quira coriacea (H. Karst.) C.C. Berg "Capinuri”" y Clarisia racemosa Ruiz \& Pav. "Mashonaste, Tulpay" (FAO 2018). Además, existen especies con uso medicinal (Mejía y Rengifo 2000, Mass y Campera 2011, Reynel et al. 2016) y alimenticio (Spichiger et al. 1990). Los frutos producidos por varias especies son indispensables para numerosas especies de frugívoros vertebrados, 
lo cual influye significativamente en la dinámica del bosque (Shanahan et al. 2001, Kanashiro 2009, Alegría 2019). Otros autores mencionan que las Moraceae se encuentran entre las diez primeras familias, en cuanto a número de especies arbóreas (Vásquez et al. 2018), siendo Pseudolmedia laevis (Ruiz \& Pav.) J.F.Macbr. la cuarta especie más abundante en la Amazonía (ter Steege et al. 2013); no obstante, Licona et al. (2007), indican que no existen muchos estudios sobre la dinámica de los bosques amazónicos y la ecología de sus especies, Asimismo, Calvi (2013) señala que uno de los principales factores que afecta la distribución y riqueza de especies de la familia Moraceae, en el Parque Nacional Madidi de Bolivia, es el estado de conservación de los bosques.

El presente estudio tuvo como objetivo conocer la composición, estructura e importancia ecológica de las Moraceae en un bosque residual de la subestación Alexander von Humboltd, del Instituto Nacional de Innovación Agraria Ucayali (Perú).

\section{Materiales y Métodos}

\section{Área de estudio}

El trabajo fue desarrollado un bosque primario residual se la subestación Alexander von Humboldt del INIA, distrito de Von Humboldt, en la provincia de Padre Abad, departamento de Ucayali (Perú), entre 849'31,7”S y $75^{\circ} 3^{\prime} 19,5^{\prime \prime} \mathrm{W}$. El sector de estudio pertenece a la Ecozona selva baja (FAO y SERFOR 2017) y fisiográficamente es de zona ondulada (perfil con ondas regulares de 5 a $10 \mathrm{~m}$ de altura) con buen drenaje (Vidaurre 1994). Presenta una precipitación estimada de $3600 \mathrm{~mm}$ y la temperatura promedio es de $26^{\circ} \mathrm{C}$ (Angulo y Fasabi 2016).

\section{Inventario de especies}

Sobre la base de cartas topográficas se definió preliminarmente el área de estudio donde, debido al grado de fragmentación del bosque, se ubicaron de manera selectiva 16 unidades de muestreo de 0,10 ha $(20 \mathrm{~m} \times 50 \mathrm{~m})$, en un rango altitudinal de 211 a $286 \mathrm{~m}$ siguiendo la metodología de Calvi (2013) (Figura 1). Se evaluó cada individuo leñoso de hábito arbóreo u hemiepífito, pertenecientes a la familia Moraceae, con diámetro a la altura del pecho (DAP) $\geq$ $2,50 \mathrm{~cm}$. Para la colecta y traslado de las muestras se empleó el protocolo de herborización de Bridson y Forman (1992).

\section{Identificación taxonómica}

Se realizó una revisión de las colecciones de Moraceae procedentes del Anexo Experimental Alexander von Humboldt, ubicadas en el Herbario Forestal del INIA-Pucallpa, así como catálogos virtuales, bibliografía especializada y bases de datos: The Plant List (http://www. theplantlist.org), Missouri Botanical Garden (http://www.tropicos.org), Global Biodiversity Information Facility-GBIF (https://www.gbif. org), NYBG Steere Herbarium (http://sweet-

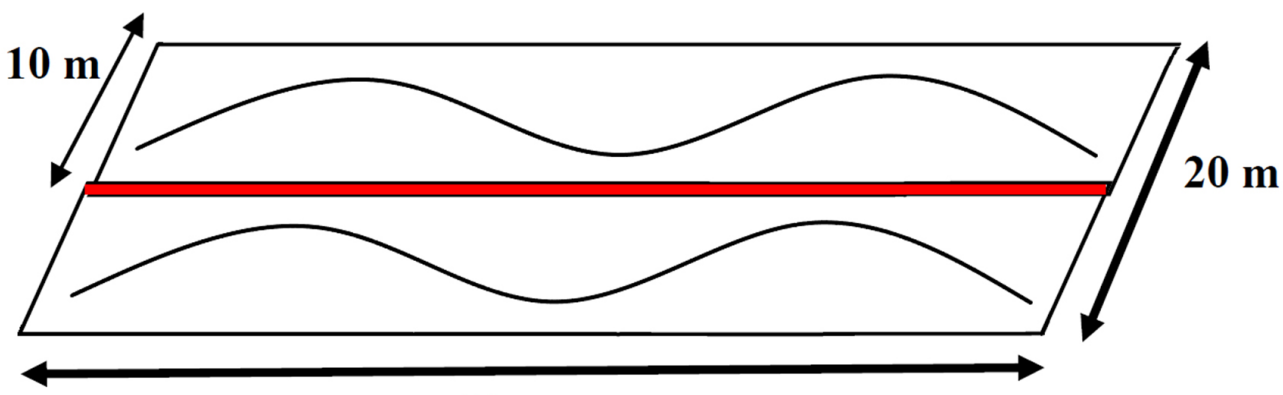

$50 \mathrm{~m}$

Figura 1. Esquema de la parcela empleada para el estudio de las Moraceae en un bosque residual. 
gum.nybg.org) y el Field Museum (https:// plantidtools.fieldmuseum.org). Posteriormente, la identificación fue corroborada en el Herbario Selva Central - Oxapampa (HOXA), estación biológica del Jardín Botánico de Missouri, ubicado en Oxapampa. Finalmente, las exsicatas fueron ingresadas en el Depositorio Biológico del INIA- Pucallpa.

\section{Análisis de datos}

Para representar la composición florística se consideraron a las especies existentes en el lugar (Lamprecht 1990, Finegan 1992, Mostacedo y Frederickzen 2000). Para calcular la eficiencia del muestreo se elaboró una curva de acumulación de especies empleando el estimador no paramétrico CHAO 2 (Colwell et al. 2005), el cual considera la distribución de las especies mediante el muestreo (Magurran 2004). El análisis se realizó mediante el programa EstimateS v.9.1.0 (Colwell 2013).

La estructura horizontal para las 16 parcelas (1,6 ha) fue representada mediante el número de individuos por clase diamétrica y la abundancia de las especies; asimismo, se calculó el área basal para conocer la distribución de la familia y dominancia de especies (Finegan 1992, Mostacedo y Frederickzen 2000, Louman et al. 2001). Los datos se agruparon en las siguientes clases diamétricas: I $(2,50$ a $9,99 \mathrm{~cm})$, II (10 a $19,99 \mathrm{~cm}$ ), III (20 a 29,99 cm), IV (30 a 39,99 $\mathrm{cm}), \mathrm{V}$ (40 a 49,99 cm), VI (50 a 59,9 cm), VII $(60$ a $69,99 \mathrm{~cm})$, VIII (70 a 79,99 cm), IX (80 a $89,99 \mathrm{~cm})$, X (90 a 99,99 cm), XI (100 a 109,99 $\mathrm{cm})$, XII (110 a 119,99 cm), XIII (120 a 120,99 $\mathrm{cm})$ y XIV (130 a 139,99 cm).

La importancia ecológica se determinó mediante el cálculo del Î́ndice de Valor de Importancia de las especies (IVI) expresado en porcentaje (Curtis y McIntosh 1951), la fórmula se representa a continuación:

Donde:

$$
\mathrm{IVI}=\mathrm{IVI}_{\mathrm{j}}=\mathrm{AbR}_{\mathrm{j}}+\mathrm{FR}_{\mathrm{j}}+\mathrm{DoR}_{\mathrm{j}}
$$

Abundancia relativa: $\mathrm{AbR}_{\mathrm{j}}=100 \times \mathrm{Ab}_{\mathrm{j}} / \sum \mathrm{Ab}_{\mathrm{j}}$

Frecuencia Relativa: $\mathrm{FR}_{\mathrm{j}}=100 \times \mathrm{F}_{\mathrm{j}} / \Sigma \mathrm{F}_{\mathrm{j}}$

Dominancia Relativa: $\operatorname{DoR}_{\mathrm{j}}=100 \times \mathrm{Do}_{\mathrm{j}} / \Sigma \mathrm{Do}_{\mathrm{j}}$
Siendo:

$A b_{j}$ : Número total de individuos de las especies $\mathrm{j}$ en todas las parcelas.

$\mathrm{F}_{\mathrm{j}}$ : Número de parcelas donde está presente la especie $\mathrm{j}$.

Do $_{\mathrm{j}}$ : Área basal total de la especie j, en todas las parcelas.

\section{Resultados}

\section{Composición florística de la familia Mora- ceae}

En las 16 parcelas de muestreo (1,6 ha) se identificaron 33 especies, en tanto que, según CHAO 2 las especies esperadas fueron 44 (Figura 2). De acuerdo con esto, la riqueza (S) observada representó el $75 \%$ de las especies que constituyen el bosque residual.

La composición de especies se distribuyó en 12 géneros: Batocarpus, Brosimum, Clarisia, Ficus, Helicostylis, Maquira, Naucleopsis, Perebea, Poulsenia, Pseudolmedia, Sorocea y Trophis. Tres géneros presentaron la mayor riqueza: $F i$ cus (8 especies), Brosimum (6 especies) y Perebea (4 especies), agrupando el 54,55\% del total de especies (Figura 3).

Cinco especies son nuevos registros para la región Ucayali: Ficus americana subsp. guianensis (Desv. ex Ham.) C.C. Berg, Ficus schultesii Dugand, Ficus ursina Standl., Ficus tonduzii Standl. vel. sp. aff., y Perebea guianensis subsp. hirsuta C.C. Berg.

\section{Estructura horizontal de la familia Moraceae}

En 1,6 ha se registraron y evaluaron 221 individuos, 138 individuos/ha a partir de $2,50 \mathrm{~cm}$ de DAP; mientras que a partir de $10 \mathrm{~cm}$ de DAP la densidad fue de 41,25 individuos/ha. El promedio del DAP, fue de $13,32 \mathrm{~cm}$ y el máximo de $136 \mathrm{~cm}$. Los individuos se agruparon en 11 de las 14 clases diamétricas consideradas para el análisis estructural, mostrando una distribución diamétrica discetánea irregular semejante a una "J" invertida (Figura 4).

Las especies con estructuras discetáneas completas fueron Pseudolmedia laevis (Ruiz 


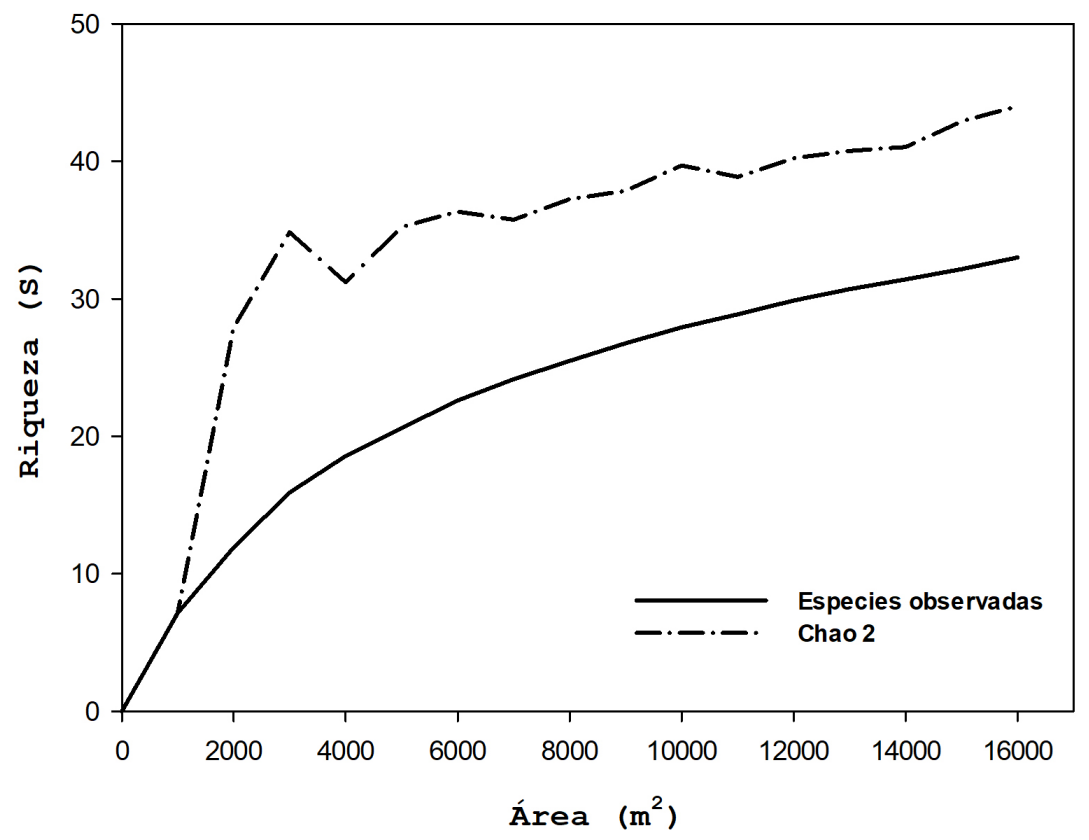

Figura 2. Curva de acumulación de especies observadas y esperadas, de la familia Moraceae en un bosque residual

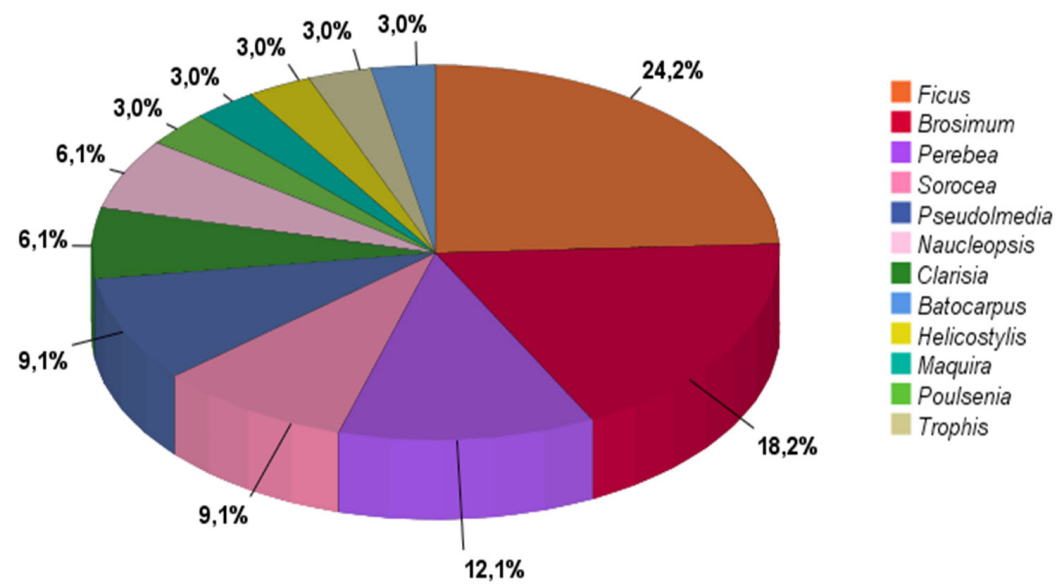

Figura 3. Riqueza de los géneros de la familia Moraceae en un bosque residual. 


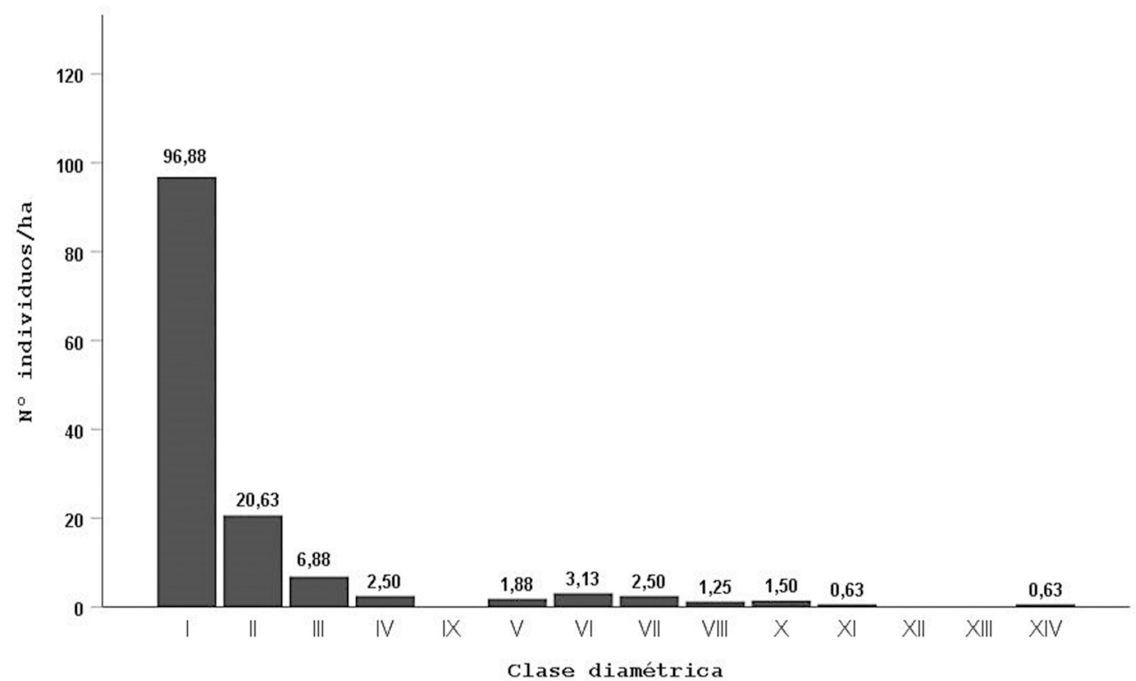

Figura 4. Estructura horizontal tipo jota invertida irregular, de las especies de la familia Moraceae en un bosque residual.

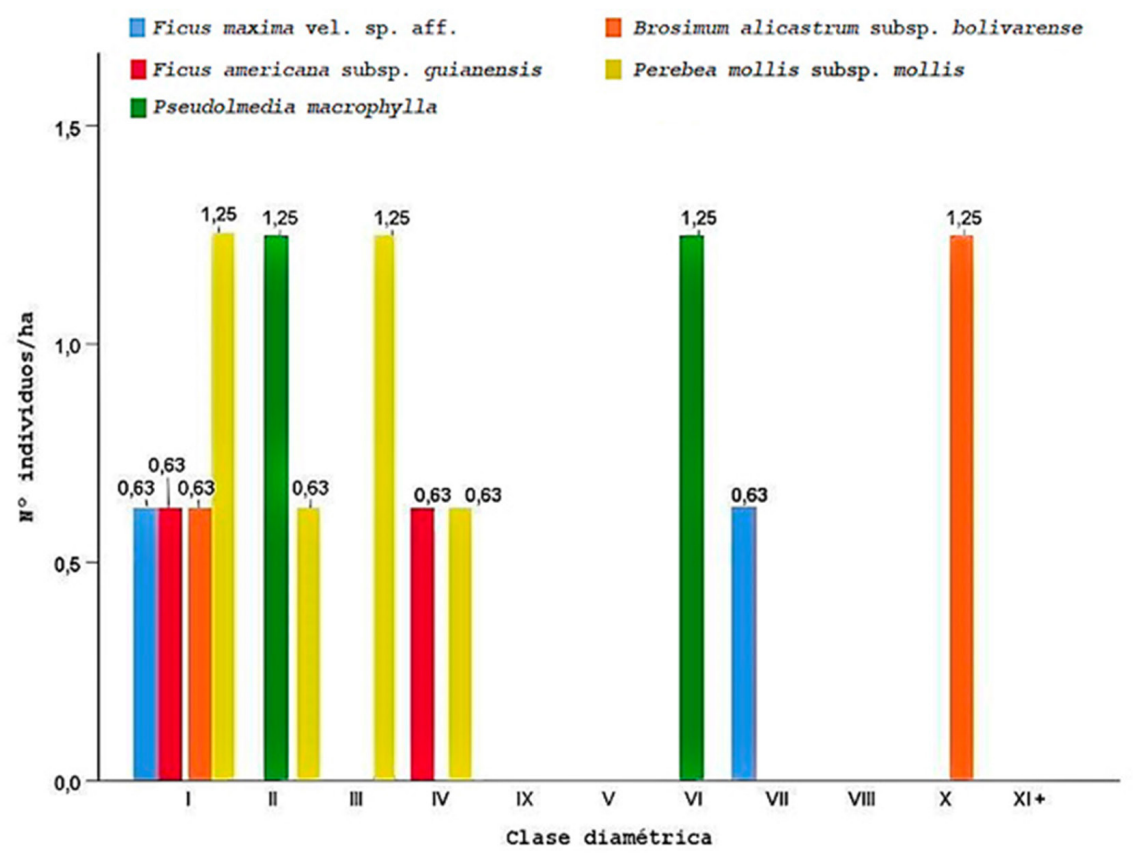

Figura 5. Especies de la familia Moraceae con estructuras horizontales bimodales, en un bosque residual. 
\& Pav.) J.F. Macbr. y Poulsenia armata (Miq.) Standl.; mientras que Brosimum lactescens (S. Moore) C.C. Berg, Clarisia biflora Ruiz \& Pav., Clarisia racemosa Ruiz \& Pav., Brosimum acutifolium subsp. obovatum (Ducke) C.C. Berg, Brosimum multinervium C.C. Berg y Brosimum utile (Kunth) Oken mostraron estructuras discetáneas irregulares. Por otro lado, Ficus maxima Mill. vel. sp. aff., F. americana sbsp. guianensis, Pseudolmedia macrophylla Trécul, Brosimum alicastrum subsp. bolivarense (Pittier) C.C. Berg y Perebea mollis subsp. mollis presentaron estructuras bimodales (Figura 5).

Otro grupo de especies se distribuyeron solo en las clases I y II. Helicostylis tomentosa (Poepp. \& Endl.) Rusby, Perebea angustifolia (Poepp. \& Endl.) C.C. Berg y Pseudolmedia laevigata Trécul presentaron estructuras horizontales de apariencias discetáneas; mientras que, Brosimum guianense (Aubl.) Huber y $\mathrm{Ba}$ tocarpus costaricensis Standl. \& L.O. Williams mostraron estructuras horizontales de apariencias bimodales.

\section{Abundancia de especies}

Las seis especies más abundantes fueron: Pseudolmedia laevis (41,88 individuos/ha),
Brosimum utile (11,25 individuos/ha), Clarisia biflora (8,75 individuos/ha), Poulsenia armata (8,75 individuos/ha) y C. racemosa $(6,25$ individuos/ha), representando el 55,7\% del total de individuos.

Las especies que estuvieron presentes solo en la clase I y fueron representadas por un solo individuo (0,63 individuo/ha) fueron: Ficus tonduzii vel. sp. aff., Ficus paraensis (Miq.) Miq., Perebea guianensis subsp. hirsuta, Naucleopsis glabra Spruce ex Pittier y Sorocea briquetii J.F. Macbr. Mientras que Perebea longipedunculata C.C. Berg, Sorocea steinbachii C.C. Berg, Naucleopsis ulei (Warb.) Ducke y Maquira calophylla (Poepp. \& Endl.) C.C. Berg presentaron $1,88,2,50,4,38$ y 5,63 individuos/ha en la clase I, respectivamente. Por otra parte, F. ursina y $F$. schultesii se distribuyeron en las clases III y VI, con 0,63 individuos/ha cada uno; en cambio, Ficus insipida subsp. insipida y Ficus popenoei Standl. presentaron 0,63 individuos/ha cada uno en la clase VII.

\section{Área basal}

El área basal total de las especies evaluadas fue de $5,81 \mathrm{~m}^{2} /$ ha. La distribución, por clase diamétrica de las especies, mostró un aumento

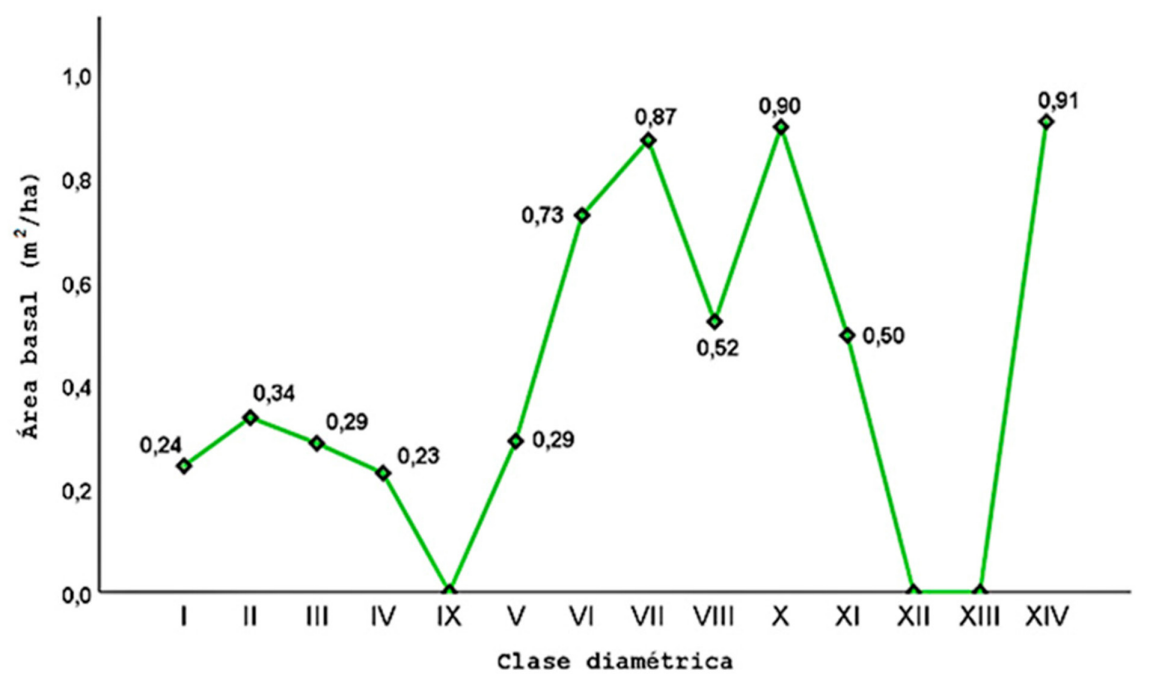

Figura 6. Distribución acumulada de las áreas basales por clase diamétrica, de la familia Moraceae en un bosque residual. 


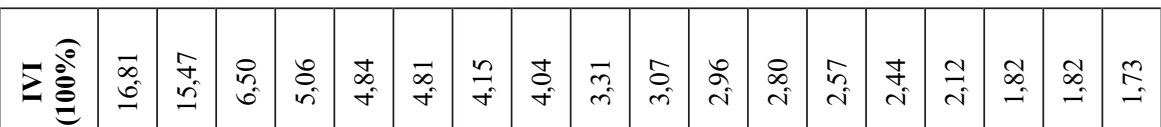

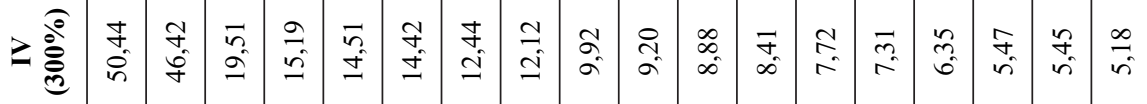

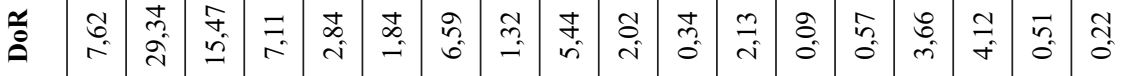

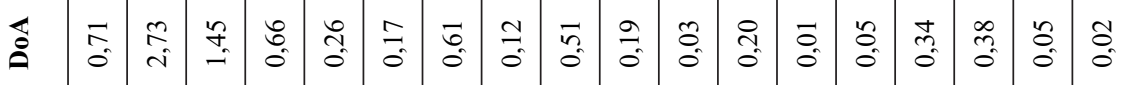

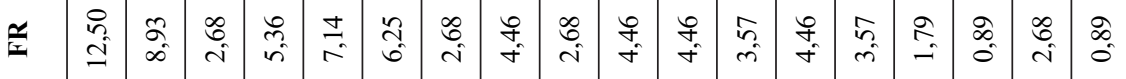

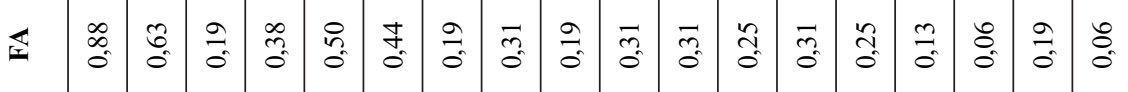

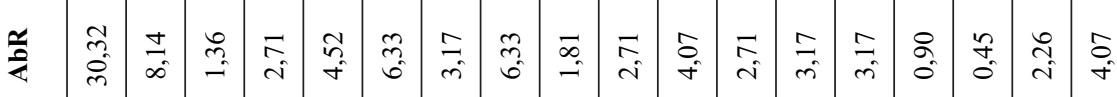

逭实

厌+

犬ิ독

売茪

. 병

봉

卷

증

\& 8

홀

के

氜

큘

这范

还要

폴

ญ.

행ํํ

ชํ요

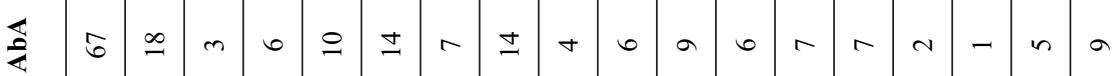

寻

ฮี 


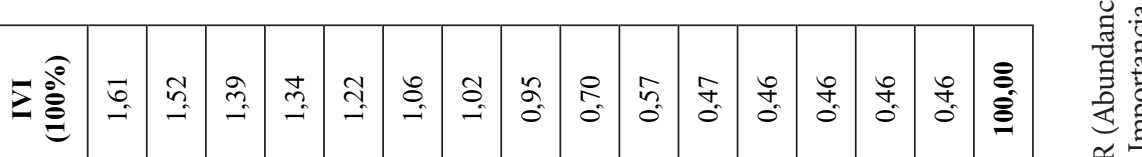

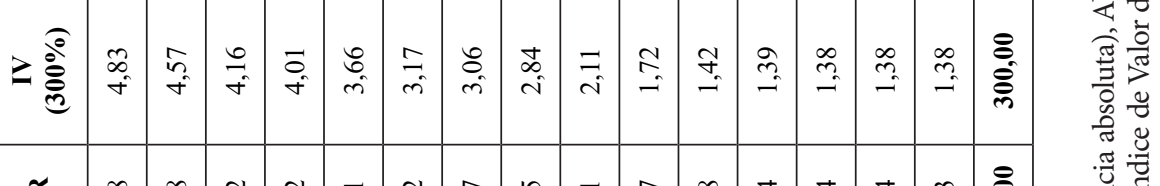

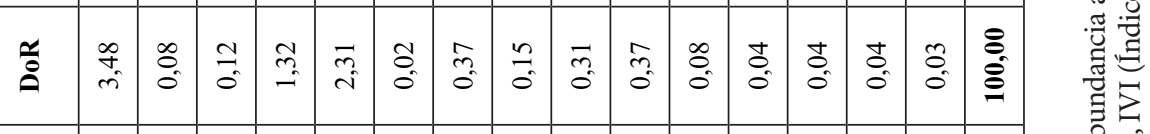

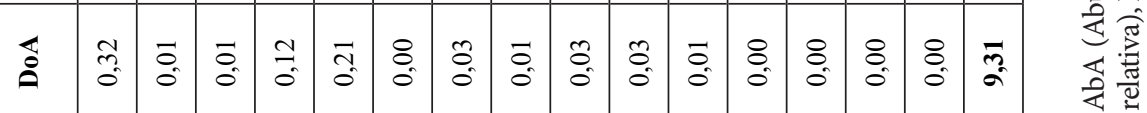

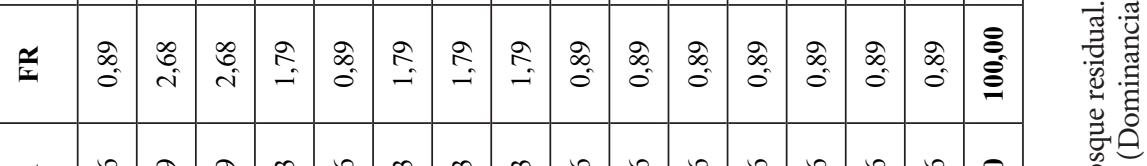

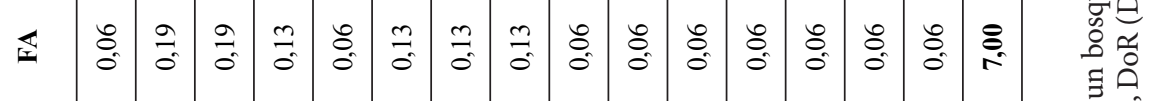

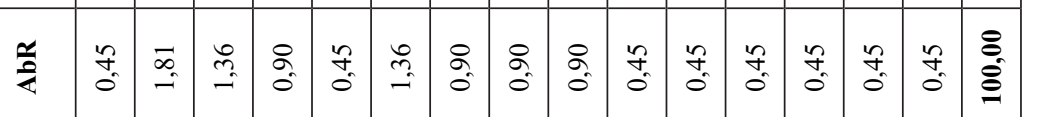
ปี

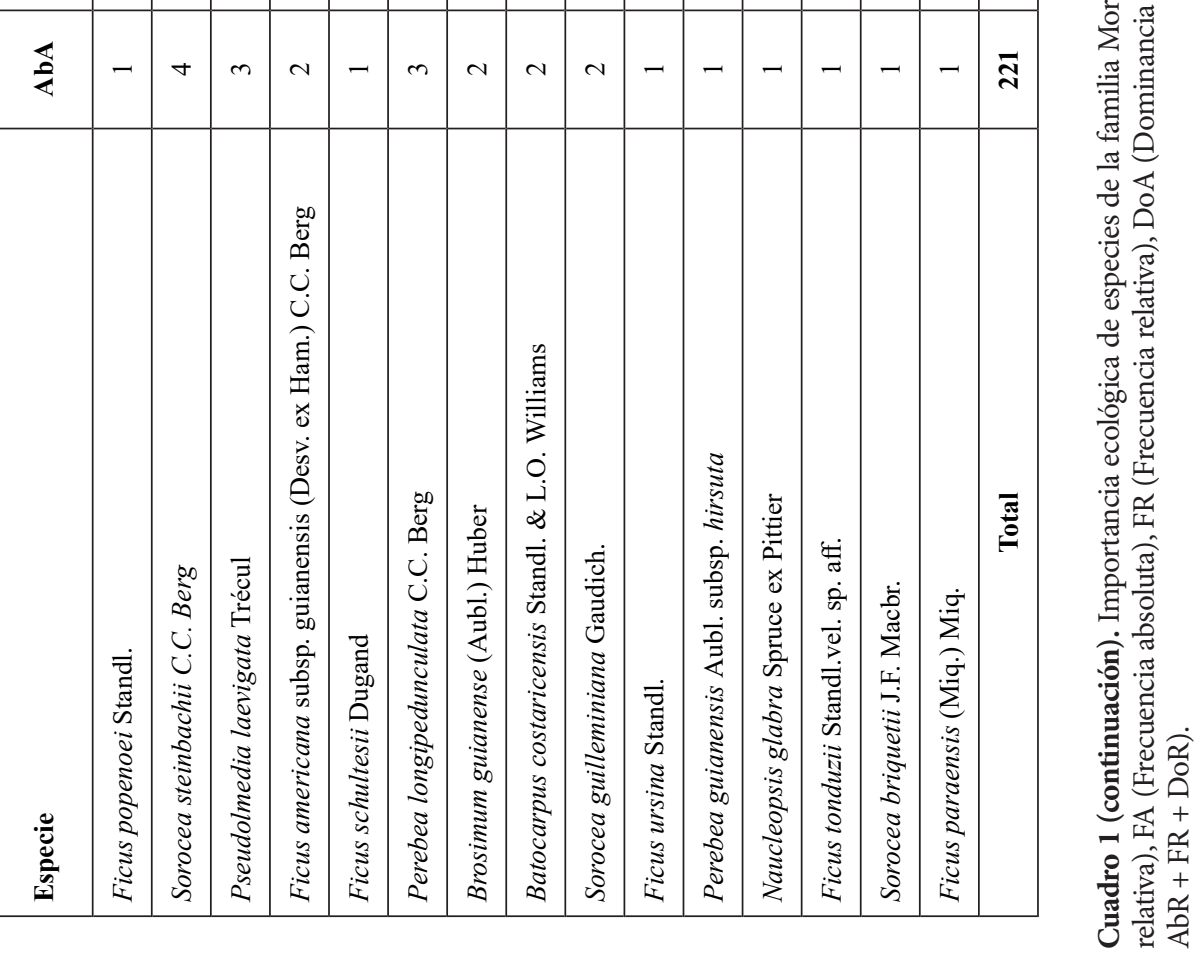


discontinuo en las últimas clases (Figura 6). El género Brosimum representó el $61,1 \%$ del total de área basal $\left(3,55 \mathrm{~m}^{2} / \mathrm{ha}\right)$. Se encontró una codominancia de especies, el primero fue Brosimum utile con $1,71 \mathrm{~m}^{2} /$ ha y el segundo fue B. alicastrum subsp. bolivarense con $0,89 \mathrm{~m}^{2} /$ ha, pese a estar representado por un solo individuo en la clase I y otro en la clase X. Ambas especies representaron el $44.80 \%$ del total área basal.

\section{Importancia ecológica de las especies de la familia Moraceae}

El peso ecológico de las seis primeras especies representó el 53,49\% del IVI. Las más importantes fueron Pseudolmedia laevis y Brosimum utile, la primera debido a su abundancia $(30,32)$ y frecuencia $(12,50)$; mientras que, la segunda por su abundancia $(8,14)$ y dominancia $(29,34)$. Las especies $B$. alicastrum subsp. bolivarense y $B$. acutifolium subsp. obovatum también fueron importantes, pero debido a su dominancia $(15,47)$ y $(7,11)$, respectivamente; mientras que, Clarisia racemosa y C. biflora, lo fueron debido a su abundancia $(4,52)$ y frecuencia $(6,25)$ (Cuadro 1).

\section{Discusión}

\section{Composición florística}

Al estudiar la composición de las Moraceae, en el bosque de Madidi en Bolivia, entre los 100 a $250 \mathrm{~m}$ de altitud, utilizando parcelas de $20 \mathrm{~m} \times 50 \mathrm{~m}$ y $10 \mathrm{~m} \times 100 \mathrm{~m}$, con un área de muestreo de 4,9 ha se encontraron 24 especies y 11 géneros (Calvi 2013), siendo esta riqueza inferior a la obtenida en el presente estudio, 33 especies y 12 géneros en 1,6 ha en un bosque primario residual. Al igual que en las investigaciones de Calvi (2013) y Marcelo-Peña y Reynel (2014), Ficus fue el género que reportó la mayor riqueza florística.

\section{Estructura horizontal}

Los bosques primarios intervenidos presentan muchas veces estructuras discetáneas irregulares, según Louman et al. (2001), esto ocurre debido a que algunas clases diamétricas tienen pocos o muchos individuos. Sin embargo, este tipo de distribución mostró que el bosque cuenta con buena reserva de individuos pequeños, a nivel de familia, en la clase I, 70\% del total de individuos y el $85 \%$ de las especies, lo cual es suficientemente abundante como para sustituir a individuos grandes. Lamprecht (1990) menciona que, la distribución antes mencionada, garantiza el rendimiento sostenible de los bosques tropicales húmedos, por lo que se puede afirmar que el aprovechamiento de todas o la mayoría de las especies de la familia Moraceae, del bosque bajo estudio, podría realizarse cumpliendo con la dimensión ecológica del manejo forestal sostenible. Pseudolmedia laevis fue la especie más abundante, concordando de este modo con ter Steege et al. (2013).

Las estructuras discetáneas completas de Pseudolmedia laevis y Poulsenia armata indican que estas especies no presentarán problemas para regenerarse; en tanto que, las especies con estructuras discetáneas irregulares (como Brosimum lactescens, Clarisia biflora, C. racemosa, $B$. acutifolium subsp. obovatum, B. multinervium y $B$. utile) y con estructuras bimodales (como Ficus maxima vel. sp. aff., F. americana sbsp. guianensis, Pseudolmedia macrophylla, Brosimum alicastrum subsp. bolivarense y Perebea mollis subsp. mollis) necesitarán de claros grandes para regenerarse (Louman et al. 2001)

El aumento discontinuo del área basal de las Moraceae en las últimas clases refleja el grado de intervención de la zona de estudio. Según Louman et al. (2001), los bosques no intervenidos, generalmente, muestran una acumulación del área basal en la última clase. Orozco y Brumér (2002), explican que si una especie tiene la mayor área basal de un sitio está dominando, aun si no es abundante, como fueron los casos de Brosimum utile y B. alicastrum subsp. bolivarense.

\section{Importancia ecológica}

Brosimum utile fue la segunda especie de mayor peso ecológico, después de Pseudolmedia laevis, obteniéndose un resultado similar al estudio realizado por Mena-Mosquera et al. (2020). Licona et al. (2007), señalan que no ex- 
iste mucha información sobre la dinámica de los bosques amazónicos y la ecología de sus especies. Por otra parte, desconocemos otros procesos biológicos asociados a la riqueza y diversidad, como los efectos provocados por los dispersores y la competencia entre plantas, que podrían ayudar a comprender muchos conceptos.

\section{Conclusiones}

El bosque residual, de la subestación Alexander von Humboldt del INIA, alberga una importante riqueza de especies de la familia Moraceae, con cinco reportes nuevos para Ucayali.

Existen diferentes estructuras horizontales entre especies pertenecientes a la misma familia, con notorias implicancias para la identificación y planificación de las intervenciones silviculturales; sugiriéndose para este tipo de bosque, que el manejo sea diversificado (o de uso múltiple) en cuanto a la utilización de las especies y la generación de bienes maderables y no maderables.

Las diferencias en la estructura e importancia ecológica son una manifestación de la individualidad de cada especie; no obstante, Pseudolmedia laevis y Brosimum utile son de resaltar ya que presentaron los mayores pesos ecológicos.

\section{Agradecimientos}

Nuestro agradecimiento a la Estación Experimental Agraria Pucallpa del INIA, por permitir desarrollar esta investigación al interior del Anexo Experimental Alexander von Humboldt y a la Direccion de Gestion Forestal y Fauna Silvestre Ucayali (DIGFFS), por autorizar el permiso de colecta. Al Herbario HOXA del Jardín Botánico Missouri y al Ing. Rodolfo Vásquez, por apoyar en la identificación de las especies. A los colegas Malhi Tarazona, Franklin Tapullima, Claudio Rodríguez, Alex Álvarez y al Téc. Ramón Pacaya por el apoyo en el levantamiento de información de campo.

\section{Referencias}

Alegría, DO. 2019. Influencia de la disponibilidad de frutos (familia Moraceae) en las dinámicas de fisión-fusión de Ateles chamek (Humboldt, 1812) en el Parque Nacional de Manu. Tesis Lic. Lima, Perú, UNALM. 75 p.

Angulo, W; Fasabi, H. 2016. Fenología de 10 especies forestales para determinar la influencia del cambio climático por efecto del calentamiento global: cinco años de estudio (20122016). Pucallpa, Perú, INIA. 31 p.

Araujo-Murakami, A; Bascopé, F; Cardona, V; Quintana, D de la; Fuentes, A; Jørgensen, P; Maldonado, K; Miranda, T; Paniagua, N; Seidel, R. 2005. Composición florística y estructura del bosque amazónico preandino en el sector del Arroyo Negro, Parque Nacional Madidi, Bolivia. Ecología en Bolivia 40(3): 281-303

Berg, C; Homeier, J. 2010. Three new species of South American Moraceae. Blumea 55: 196-200. DOI: https://doi. org/10.3767/000651910X527707.

Brako, L; Zarucchi, J. 1993. Catálogo de las Angiospermas y Gimnospermas del Perú. Monogr. Syst. Bot. Missouri Bot. Garden. 45: 1-1286.

Bridson D; Forman, L. 1992. The Herbarium Handbook. Kew, Reino Unido, Royal Botanic Gardens, Kew. United Kingdom. 93 p.

Calvi, SP. 2013. Diversidad y distribución de la familia Moraceae en los bosques de la Región Madidi, La Paz, Bolivia. Tesis Lic. La Paz, Bolivia, Universidad Mayor de San Andrés. 67 p.

Cardona, V; Fuentes, A; Cayola, L. 2005. Las moráceas de la región de Madidi, Bolivia. Ecología en Bolivia 40(3): 212-264.

Colwell, RK. 2013. EstimateS 9.1.0 user's guide: Statistical estimation of species richness and shared species from samples. Connectitut, Estados Unidos de América, University of Connecticut.

Colwell, RK; Xuan Mao, C; Chang, J. 2005. Interpolando, extrapolando y comparando las curvas de acumulación de especies basadas en su incidencia. Ecology 85(10): 2717-2727. 
Curtis, JT; McIntosh, RP. 1951. An upland forest continuum in the prairie- forest border region of Wisconsin. Ecological Society of America 32(3): 476-496. DOI: https://doi. org./10.2307/1931725.

FAO (Naciones Unidas para la Alimentación y la Agricultura, Italia); SERFOR (Servicio $\mathrm{Na}-$ cional Forestal y de Fauna Silvestre, Perú). 2017. Nuestros bosques en números: primer reporte del Inventario Nacional Forestal y de Fauna Silvestre (en línea). Lima, Perú. Consultado 28 jun. 2017. Disponible en https://sinia.minam. gob.pe/documentos/nuestros-bosques-numeros.

FAO (Organización de las Naciones Unidas para la Alimentación y la Agricultura). 2018. La Industria de la Madera en el Perú: Identificación de las barreras y oportunidades para el comercio interno de productos responsables de madera, provenientes de fuentes sostenibles y legales, en las MIPYMEs del Perú. Lima, Perú, CITEmadera/FAO. 178 p.

Finegan, B. 1992. El potencial de manejo de los bosques húmedos secundarios neotropicales de las tierras bajas. Luján $\mathrm{R}$ (trad.). Turrialba, Costa Rica, CATIE. 30 p. (Colección Silvicultura y manejo de bosques naturales, $\left.\mathrm{n}^{\circ} .5\right)$.

Flores, Y. 2018. Árboles nativos de la región Ucayali, Perú. Pucallpa, Perú. INIA. 439 p.

García, C; Marín, H; Moriones, D; Muñoz, M; Valencia, C. 2014. Estructura, composición y diversidad de los Bosques Naturales De Smurfit Kappa Cartón de Colombia: Popayán y Cajibío. Biotecnología en el Sector Agropecuario y Agroindustrial 12(1): 10-19.

García, WO. 2019. Composición florística de la familia Moraceae, como fuente de carbono aéreo en la gradiente altitudinal de un bosque siempreverde, piemontano de la amazonia Ecuatoriana, año 2018. Tesis MSc. Quevedo, Ecuador, UTEQ. 95 p.

Honorio, E; Reynel, C. 2003. Vacíos de colección de la flora de los bosques húmedos del Perú. Lima, Herbario Facultad de Ciencias Forestales-UNALM. 87 p.
Huamantupa, I; Vásquez, R; Foster, R; Cuba, M; Calatayud, G. 2014. Adiciones de angiospermas a la flora del Perú procedentes de los bosques Andino Amazónicos del sur peruano. Revista peruana de biología 21(2): 163-170. DOI: https://doi.org/10.15381/rpb.v21i2.9819.

Kanashiro, LJ. 2009. Etología de Forrajeo de Ateles belzebuth chamek (Atelidae: Atelinae) en el Parque Nacional del Manu durante la temporada seca 2005. Tesis Lic. Lima, Perú, UNAL.

Lamprecht, H. 1990. Silvicultura en los Trópicos. Carrillo, A (trad.). Eschborn, República Federal de Alemania, GTZ. 335 p.

Licona, JC; Peña, M; Mostacedo, B. 2007. Composición florística, estructura y dinámica de un bosque amazónico aprovechado a diferentes intensidades en Pando, Bolivia. Santa Cruz, Bolivia. $60 \mathrm{p}$.

Louman, B; Quirós, D; Nilsson, M. 2001. Silvicultura de bosques latifoliados húmedos con énfasis en América Central. Turrialba, Costa Rica, CaTIE. 265 p. (Manual técnico n 46).

Magurran, EA. 2004. Measuring biological diversity. Oxford, Reino Unido, Blackwell Publishing. $256 \mathrm{p}$.

Marcelo-Peña, JL; Reynel, C. 2014. Patrones de diversidad y composición florística de parcelas de evaluación permanente en la selva central de Perú. Rodriquésia 65(1): 35-47. DOI: https:// doi.org/ 10.1590/S2175-78602014000100003.

Mass, W; Campera, M. 2011. Árboles medicinales: conocimientos y usos en la cuenca baja del río Marañón, zona de amortiguamiento de la Reserva Nacional Pacaya Samiria. Iquitos, Perú, MINAM. 81 p.

Mejía, K; Rengifo, E. 2000. Plantas medicinales de uso popular en la Amazonía Peruana. 2 ed. Lima, Perú, IIAP. 286 p.

Mena-Mosquera, VE; Andrade, HJ; TorresTorre, JJ. 2020. Composición florística, estructura y diversidad del bosque pluvial tropical de la subcuenca del río Munguidó, Quibdó, Chocó, Colombia. Entramado 16: 204-215. DOI: https://dx.doi.org/10.18041/1900-3803/ entramado.1.6109. 
MINAM (Ministerio del Ambiente, Perú). 2014. Estrategia nacional de diversidad biológica al 2021: plan de acción 2014 - 2018. Lima, Perú. 114 p.

Mitidieri, N; Cardoso, L; Damián, A; Albán, J. 2020. A new species and a new record of Ficus sect. Pharmacosycea (Moraceae) from Peru. Systematic Botany 45(1): 91-95. DOI: https:// doi.org/10.1600/036364420X15801369352342.

Mostacedo, B; Fredericksen, T. 2000. Manual de métodos básicos de muestreo y análisis en ecología vegetal. Santa Cruz de la Sierra, Bolivia, BOLFOR. 87 p.

Mostacedo, B; Balcazar, J; Montero, JC. 2006. Tipos de bosque, diversidad y composición florística en la Amazonia sudoeste de Bolivia. Ecología en Bolivia. 41(2): 99-116.

Nebel, G; Dragsted, J; Vanclay, JK. 2000. Estructura y composición florística del bosque de la llanura aluvial inundable de la Amazonía Peruana: II El sotobosque de la restinga. Folia Amazonia 10 (1-2): 151-181. DOI: https://doi. org/10.24841/fa.v10i1-2.246.

Neill, D; Killeen, T. 1991. Curso de dendrología tropical en la Amazonía boliviana, Valle de Sacta. La Paz, Bolivia, Herbario Nacional de Bolivia. $60 \mathrm{p}$.

Orozco, L; Brumér, L. 2002. Inventarios forestales para bosques latifoliados en América Central. Turrialba, Costa Rica, CATIE. 264 p.

Reynel, C; Pennington, TD; Pennington, RT. 2016. Árboles del Perú. Lima, Perú, Imprenta Bellido. 1047 p.

Shanahan, M; Samson, SO; Estephen, SG; Corlett, R. 2001. Fig-eating by vertebrate frugivores: a global review. Biological Reviews 76(4): 529-572. DOI: https://doi. org/10.1017/S146479310100576010.1017/ $\underline{\mathrm{S} 1464793101005760 .}$.

SERFOR (Servicio Nacional Forestal y de Fauna Silvestre, Perú). 2020. Anuario Forestal y de Fauna Silvestre 2019. Lima, Perú. 132 p.

Spichiger, R; Méroz, J; Loizeau, P; Stutz, L. 1990. Contribución a la flora de la Amazonía Peru- ana: árboles del Arborétum Jenaro Herrera. Ginebra, Suiza, IIAP. 359 p.

ter Steege, H; Pitman, N; Sabatier, D; Baraloto, $\mathrm{CH}$; Salomão,R; Guevara, JE; Phillips, O; Castilho, C; Magnusson, W; Molino, J; Monteagudo, A; Núñez, P; Montero, JC; Feldpausch, T; Honorio, EH; Killeen, TJ; Mostacedo, B; Vasquez, R; Assis, RL; Terborgh, J; Wittmann, F; Andrade, A; Laurance, WF; Laurance, SG; Marimon, BS; Marimon, BH; Guimarães, IM; Leão, I; Brienen, R; Castellanos, H; Cárdenas, D; Duivenvoorden, JF; Mogollón, HF; Dionízia, F; Dávila, N; García-Villacorta, R; Stevenson, PR; Costa, F; Emilio, T; Levis, C; Schietti, J; Souza, P; Alonso, A; Dallmeier, F; Duque, AJ; Fernandez, MT; Araujo-Murakami, A; Arroyo, L; Gribel, R; Fine, R; Peres, C; Toledo, M; Aymard, GA; Baker, TR; Cerón, C; Engel, J; Henkel, TW; Maas, P; Petronelli, P; Stropp, J; Eugene, Cha; Daly, D; Neill, D; Silveira,M; Paredes, M; Chave, J; Lima, D; Møller, P; Fuentes, A; Schöngart, J; Cornejo, F; Di Fiore, A; Jimenez, EM; Peñuela, MC; Fernando, J; Rivas, G; Van, T; Von, P; Hoffman, B; Zent, E; Malhi, Y; Prieto, A; Rudas, A; Ruschell, AR; Silva, N; Vos, V; Zent, S; Oliveira, AA; Cano, A; Gonzales, T; Nascimento, T; Ramirez, I, Sierra, R; Tirado, M; Umaña, MN; Heijden, G; Vela, CI; Vilanova, E; Vriesendorp, C; Wang, O; Young, K; Baider, C; Balslev, H; Ferreira, C; Mesones, I; Torres, A; Urrego, LE; Zagt, R; Alexiades, MN; Hernandez, L; Huamantupa, I; Milliken, W; Palacios, W; Pauletto, D; Valderrama, E; Valenzuela, L; Dexter, KG; Feeley, K; Lopez, G; Silman, MR. 2013. Hyperdominance in the Amazonian tree flora. Science (342): 245-337. DOI: https://doi. org/10.1126/science.1243092.

Ulloa Ulloa C; Zarucchi, JL; León, B. 2004. Diez años de Adiciones a la Flora del Perú: 19932003. Arnaldoa (Edic. Esp. Noviembre 2004): 1-242.

Ulloa Ulloa C.; Acevedo-Rodríguez, P; Beck, S; Belgrano, M; Bernal, R; Berry, P; Brako, L; Celis, M; Davidse, G; Forzza, R; Gradstein, R; Hokche, O; León, B; León-Yáñez, S; Magill, R; Neill, D; Nee, M; Raven, P; Stimmel, H; Strong, M; Villaseñor, J; Zarucchi, J; Zuloaga, F; Jorgensen. 2017. An integrated assessment of the 
vascular plant species of the Americas. Science 358(6370): 1614-1617. DOI: https://doi. org/10.1126/science.aao0398.

Vásquez, R; Rojas, R; Monteagudo, A; Valenzuela, L; Huamantupa, I. 2018. Catálogo de árboles del Perú. Revista Q’euña 9(1): 1-167.

Vidaurre, H. 1994. Balance de experiencias silviculturales con Cedrelinga cataeniformis Ducke (Mimosoideae) en la Región de Pucallpa, Amazonía Peruana. Tesis MSc. Turrialba, Costa Rica, CATIE. 165 p. 